\title{
Distributing Points on the Sphere, I
}

\author{
Ali Katanforoush and Mehrdad Shahshahani
}

\section{CONTENTS}

1. Introduction

2. The Metrics

3. The Methods

4. Numerical Results

References

Acknowledgments
2000 AMS Subject Classification: Primary 52C35;

Secondary 31-04

Keywords: Distributing points on a sphere, Fekete points, logarithmic potential, Columb potential
We study four different methods for distributing points on the sphere and numerically analyze their relative merits with respect to certain metrics.

\section{INTRODUCTION}

The problem of "evenly" distributing points on a sphere has a long history. Albeit its intuitive meaning, it is necessary to define "even distribution" mathematically. Various metrics, $\vartheta_{s}, 0 \leq s \leq \infty$ (see below for definitions), whose extrema may correspond to even distributions have been proposed. The starting point of this study is Problem 7 in [Smale 00] where the implications of the distribution of points on the sphere, corresponding to the global minimum of $\vartheta_{\circ}$, for numerical analysis are discussed. A different version of the problem based on minimizing the Coulomb potential of electrons distributed on a sphere is generally known as the Thomson problem in spite of the fact that the problems considered in [Thomson 04] are quite different. [Altschuler et al. 97] reports on a numerical study of Thomson's problem where points are distributed on the sphere according to a number theoretic algorithm. In [Sarnak 90], the issue of constructing $\epsilon$-good sets $\left\{R_{1}, \cdots, R_{n}\right\} \subset S O(3)$ and its relation to the Ruziewicz problem is investigated. The explicit construction in [Sarnak 90] gives a method for distributing points on $S^{2}$ by applying $R_{j}$ s (or words in $R_{j} \mathrm{~s}$ ) to a random point in $S^{2}$. In [Rakhmanov et al. 94] and [Kuijlaars and Saff 98], various notions of energy (or metric) for points on a sphere and some theoretical results are discussed.

In this work, we numerically analyze four different methods for distributing points on $S^{2}$. Besides the methods given in [Altschuler et al. 97] and [Sarnak 90], we discuss two simple geometric algorithms, the "subdivision" and "polar coordinates" methods, the details of which appear in the next section. The analysis of the merits of the algorithms is based on the calculation of the metrics $\vartheta_{\circ}$, and $\vartheta_{1}$, and we will make some remarks about 
$\vartheta_{2}$ and $\vartheta_{\infty}$. Given points $z_{1}, \ldots, z_{N}$ on the sphere $S^{2}$ of unit radius, we set

$$
\vartheta_{s}=\vartheta_{s}\left(z_{1}, \ldots, z_{N}\right)=\sum_{1 \leq j<k \leq N} \frac{1}{\left|z_{j}-z_{k}\right|^{s}},
$$

where $s>0$ is a positive real number and $\left|z_{j}-z_{k}\right|$ denotes the distance in $\mathbb{R}^{3}$ of $z_{j}$ and $z_{k} . \vartheta_{\infty}$ is defined as

$$
\vartheta_{\infty}=\vartheta_{\infty}\left(z_{1}, \ldots, z_{N}\right)=\min _{1 \leq j<k \leq N}\left|z_{j}-z_{k}\right| .
$$

The metric $\vartheta_{\circ}=\vartheta_{\circ}\left(z_{1}, \ldots, z_{N}\right)$ is more appropriately defined for points on the sphere $S_{\frac{1}{2}}^{2}$ of unit diameter and is defined as

$$
\vartheta_{\circ}=\sum_{1 \leq j<k \leq N} \log \left[\frac{1}{\left|z_{j}-z_{k}\right|}\right] .
$$

Here also, $\left|z_{j}-z_{k}\right|$ refers to the distance of $z_{j}$ and $z_{k}$ in $\mathbb{R}^{3}$.

The numerical evidence reported in this article suggests that the simpler geometric methods give values for $\vartheta_{\circ}$ and $\vartheta_{1}$ which are closer to the actual optima than those obtained in [Altschuler et al. 97] (lattice point method) or [Sarnak 90]. In fact, we obtain configurations, via the polar coordinates method, for which $\vartheta_{1}$ is smaller than the conjectured global minimum reported in [Altschuler et al. 97]. The performance of the method of [Sarnak 90] in decisively inferior to the lattice point or the polar coordinates methods for $\vartheta_{s}, s \in[0, \infty]$. For a small or moderate number of points, standard optimization algorithms give only slight improvements over the values reported here. The fact that the optimized values for $\vartheta_{\circ}\left(\right.$ or $\left.\vartheta_{1}\right)$ are close is a reflection of the profusion of local minima for either metric. For a large number of points (more than 5,000), the computationally intensive nature of the optimization algorithms render them impractical.

A disadvantage of the method in [Altschuler et al. 97] is that to distribute $N$ points on $S^{2}, N$ should satisfy a certain diophantine equation which has a solution only for a small fraction of positive integers $N$. The polar coordinates method, on the other hand, is applicable to practically every $N$. One may optimistically conjecture that the polar coordinates method will distribute points on the sphere in such a way that the estimate,

$$
\left|\vartheta_{\circ}\left(z_{1}, \ldots, z_{N}\right)+\left(\begin{array}{c}
N \\
2
\end{array}\right) \log V_{N}\right| \leq C \log N,
$$

of Problem 7 of [Smale 00] is fulfilled for $N \leq 10^{5}$ (see Section 3 for a definition of $V_{N}$ and explanations) with $C \leq 2$.
In the application of any optimization algorithm to a complex problem, the judicious choice of an initial point is critical. The emphasis of this paper is the choice of a "good" initial point. A successful application of an optimization algorithm via the application of the fast multipole method (see, e.g., [Greengard and Rokhlin 97]) may be possible. This issue is currently under investigation and is not discussed here. It should be pointed out that a random choice of points on $S^{2}$ (according to the standard invariant measure) leads to poor values for $\vartheta_{s}$ as compared to those obtained via the algorithm in [Altschuler et al. 97] and a fortiori to the polar coordinates method.

\section{THE METRICS}

The motivation for the metric $\vartheta_{1}$ is the familiar Coulomb potential. Minimizing the metric $\vartheta_{1}$ appears to have implications for the stable molecular configurations in chemistry. We set

$$
\mathcal{E}_{N}^{s}=\inf \vartheta_{s}\left(z_{1}, \ldots, z_{N}\right), \text { for } s<\infty,
$$

where inf (or, in fact, $\min$ ) is taken relative to all possible configurations of $N$ points on $S^{2}$. For $s=\infty$, we set

$$
\mathcal{E}_{N}^{\infty}=\sup \vartheta_{\infty}\left(z_{1}, \ldots, z_{N}\right) .
$$

It is known $[\mathrm{KS}]$ that $\mathcal{E}_{N}^{s}$ admits of the asymptotic expansion

$$
\mathcal{E}_{N}^{s} \simeq \frac{2^{-s}}{2-s} N^{2}+O\left(N^{1+\frac{s}{2}}\right), \text { for } 0<s<2,
$$

as $N \longrightarrow \infty$. Furthermore,

$$
\lim _{N \rightarrow \infty} \frac{\mathcal{E}_{N}^{2}}{N^{2} \log N}=\frac{1}{8}
$$

For $s=0$, we have

$$
\mathcal{E}_{N}^{\circ} \simeq \frac{1}{4} N^{2}-\frac{1}{4} N \log N+O(N) .
$$

The metric $\vartheta_{\circ}$ appears in classical potential theory. Let $z_{1}, \ldots, z_{N}$ be points on the sphere $S_{\frac{1}{2}}^{2} \subset \mathbb{R}^{3}$ of diameter 1 , and let

$$
U_{N}=U_{N}\left(z_{1}, \ldots, z_{N}\right)=\left[\prod_{1 \leq i<j \leq N}\left|z_{j}-z_{k}\right|\right]^{\left.\frac{1}{(N)} 2\right)} .
$$

Set

$$
V_{N}=\sup U_{N}\left(z_{1}, \ldots, z_{N}\right)
$$


where sup is taken relative to all configurations of $N$ points. Then $V_{N+1} \leq V_{N}$ and it is a classical result (see [Tsuji 75], Chapter III) that

$$
\lim _{N \rightarrow \infty} V_{N}=\frac{1}{\sqrt{e}}
$$

compatible with (2-3). For each $N$, the supremum in the definition of $V_{N}$ is, in fact, a maximum. A set of points $\left\{z_{1}, \cdots, z_{N}\right\}$ realizing sup is called elliptic Fekete points. It is shown in [Shub and Smale 93] that for such a set $\left\{z_{1}, \cdots, z_{N}\right\}$, the polynomials

$$
P_{N}(z)=\prod_{k=1}^{N}\left(z-z_{k}\right)
$$

have desirable condition numbers. Here, $P_{N}$ is regarded as a polynomial in $z \in \mathbb{C}$ by the stereographic projection of the points $z_{k}$ to the complex plane. The condition number of a polynomial as defined in [Shub and Smale 93] is a modification of the classical definition $\sup \frac{1}{\left|P^{\prime}(\zeta)\right|}$ where sup is taken relative to the zeros of $P$. The remarkable property of Fekete points is that the condition number of $P_{N}$ is bounded by $\sqrt{N(N+1)}$, while for randomly chosen $z_{1}, \cdots, z_{N}$, the condition number of $P_{N}(z)$ appears to grow exponentially fast. The importance of slowly growing condition numbers is due to their application to the Newton and homotopy methods in numerical analysis.

While the existence of elliptic Fekete points and the limit $(2-4)$ is classical, their explicit determination is a major unsolved problem. It is therefore desirable to obtain approximations. To properly quantify the approximation, we take the logarithm of $U_{N}$ which gives

$$
-\left(\begin{array}{c}
N \\
2
\end{array}\right) \log U_{N}=\vartheta_{\circ}
$$

In view of $(2-3)$, the lead term of the asymptotic expansion of $-\left(\begin{array}{c}N \\ 2\end{array}\right) \log V_{N}$ is $\frac{N^{2}}{4}$ and, in fact, we have

$$
-\left(\begin{array}{c}
N \\
2
\end{array}\right) \log V_{N}=\frac{N^{2}}{4}-\frac{N \log N}{4}+O(N),
$$

as $N \longrightarrow \infty$. The estimate $N \log N$ for the second term follows from standard arguments of classical potential theory. It quantifies the rate of convergence of $V_{N}$ to $\frac{1}{\sqrt{e}}$ in $(2-4)$. An approximate version of the problem of the explicit determination of Fekete points is to exhibit $z_{1}, \ldots, z_{N}$ such that

$$
\left|\vartheta_{\circ}\left(z_{1}, \ldots, z_{N}\right)+\left(\begin{array}{c}
N \\
2
\end{array}\right) \log V_{N}\right| \leq C \log N .
$$

To better understand the quantities $\vartheta_{s}$, we introduce some notation

$$
\begin{aligned}
& \xi_{\circ}=\lim _{N \rightarrow \infty} \frac{\vartheta_{\circ}-\frac{N^{2}}{4}}{N \log N}, \quad \eta_{\circ}=\lim _{N \rightarrow \infty} \frac{\vartheta_{\circ}-\frac{N^{2}}{4}+\frac{N \log N}{4}}{N}, \\
& \zeta_{\circ}=\lim _{N \rightarrow \infty} \frac{\vartheta_{\circ}-\frac{N^{2}}{4}+\frac{N \log N}{4}+\eta_{\circ} N}{\log N},
\end{aligned}
$$

and

$$
\begin{aligned}
& \xi_{1}=\lim _{N \rightarrow \infty} \frac{\vartheta_{1}-\frac{N^{2}}{2}}{N^{\frac{3}{2}}}, \quad \eta_{1}=\lim _{N \rightarrow \infty} \frac{\vartheta_{1}-\frac{N^{2}}{2}+\xi_{1} N^{\frac{3}{2}}}{N}, \\
& \xi_{2}=\lim _{N \rightarrow \infty} \frac{\vartheta_{2}-\frac{1}{8} N^{2} \log N}{N^{2}} .
\end{aligned}
$$

\section{THE METHODS}

We briefly describe four algorithms for generating points on a sphere.

\section{$3.1 \epsilon$-Good Sets}

A finite set $\left\{R_{1}, \ldots, R_{n}\right\} \subset S O(3)$ is called an $\epsilon$-good set if for every continuous function $\psi$ on $S^{2}$ with $\int \psi=0$, there is $j$ such that

$$
\int_{S^{2}}\left|\psi(x)-\psi \circ R_{j}(x)\right|^{2} d x \geq \epsilon^{2} \int_{S^{2}}|\psi(x)|^{2} d x .
$$

It is an elementary argument that $\epsilon \leq \sqrt{2}$ is necessary for the existence of an $\epsilon$-good set. We make use of the algorithm for constructing $\epsilon$-good sets for arbitrary $\epsilon<$ $\sqrt{2}$ given in [Sa]. Let $p \equiv 1 \bmod 4$ be a prime. Then according to a classical theorem of Jacobi, the equation

$$
a_{\circ}^{2}+a_{1}^{2}+a_{2}^{2}+a_{3}^{2}=p
$$

has $8(p+1)$ solutions $\left(a_{\circ}, a_{1}, a_{2}, a_{3}\right)$ in integers. Each solution yields a quaternion $g_{a}=a_{\circ}+a_{1} \mathbf{i}+a_{2} \mathbf{j}+a_{3} \mathbf{k}$. The quaternion group $\mathbf{H}=\{ \pm 1, \pm \mathbf{i}, \pm \mathbf{j}, \pm \mathbf{k}\}$ of order eight acts on the set of solutions, and from each orbit, we pick one solution. Thus, we obtain $p+1$ solutions of the form

$g_{a}=a_{\circ}+a_{1} \mathbf{i}+a_{2} \mathbf{j}+a_{3} \mathbf{k}, \quad \overline{g_{a}}=a_{\circ}-a_{1} \mathbf{i}-a_{2} \mathbf{j}-a_{3} \mathbf{k}$.

Now $h_{a}=\frac{g_{a}}{\sqrt{g_{a} \overline{g_{a}}}}$ and $h_{a}^{\prime}=\frac{\overline{g_{a}}}{\sqrt{g_{a} \overline{g_{a}}}}$ lie in $S U(2)$ and are inverses to each other. The images of the unit quaternions $h_{a}, \overline{h_{a}}$ in $S O(3)$ under the canonical projection $S U(2) \rightarrow S O(3)$ give an $\epsilon$-good set $\left\{R_{1}, \ldots, R_{p+1}\right\}$. Here, $\epsilon$ depends on $p$ and by taking $p \equiv 1 \bmod 4$ sufficiently large, we can make $\epsilon$ arbitrarily close to $\sqrt{2}$. One can also take $p$ to be a small prime, and instead look at words of a given length in $R_{1}, \ldots, R_{p+1}$. In either case, 
to generate points on $S^{2}$, we consider a random point $e \in S^{2}$ and look at its image under the action of the $\epsilon$ good subset of $S O(3)$. Since for the construction of an $\epsilon$-good set, we only used one representative from each orbit of $\mathrm{H}$, the distribution of points on $S^{2}$ will be very biased and it is necessary to choose representatives from orbits under $\mathbb{Z} / 2 \simeq\{ \pm 1\}$.

\subsection{Lattice Points}

This method was used in [Altschuler et al. 97] to generate an initial configuration for $N=10\left(m^{2}+n^{2}+m n\right)+2$, where $m$ and $n$ are integers, points on $S^{2}$. By optimizing relative to the metric $\vartheta_{1}$, it was surmised that the absolute minimum was, in fact, attained provided $N$ is only moderately large (for example, $<5,000$ ). Generally speaking, the method of [Altschuler et al. 97] is as follows: Let $\zeta=e^{\frac{i \pi}{3}}$ and consider the equilateral triangle $\Delta$ in $\mathbb{C}$ with vertices at $0, m+\zeta n$ and $\zeta m+\zeta^{2} n$. The intersection of the lattice $\mathcal{L}$ generated by $1, \zeta$ with $\Delta$ is a finite number of points in $\Delta$. By mapping $\Delta$ onto a face of the regular icosahedron inscribed in the unit sphere, repeating the process by reflections for the other faces, and projecting radially onto $S^{2}$, we obtain $N$ points on $S^{2}$. The resulting configuration is the initial point for minimizing $\vartheta_{1}$ in [Altschuler et al. 97].

The diophantine equation $N=10\left(m^{2}+n^{2}+m n\right)+2$ does not have a solution for every $N$ and therefore, the method of [Altschuler et al. 97] is applicable only to those $N$ for which it has a solution. Let $\nu_{A}(N)$ be the number of integers $l \leq N$ for which $l=10\left(m^{2}+n^{2}+m n\right)+2$ has a solution. According to our calculations,

$$
\nu_{A}(N)=c_{1} N+c_{2} \sqrt{N}+o(\sqrt{N}),
$$

where $c_{1} \simeq .018$ and $c_{2} \simeq 2.1$. It is classical and easy to prove that the density of values assumed by a positive definite quadratic form $a x^{2}+b x y+c y^{2}$, as $x, y$ range over the integer lattice, is asymptotically given by

$$
\frac{2 \pi}{\sqrt{4 a c-b^{2}}} N+o(N) \text {. }
$$

However, in this formula, values are counted with multiplicity and is therefore irrelevant to the present context.

\subsection{Subdivision of the Icosahedron}

Given a triangle $A B C$, let $a, b$, and $c$ be the midpoints of the sides $B C, C A$, and $A B$, respectively. Then the triangle $a b c$ subdivides $A B C$ into four triangles. The same process can be applied to all 20 faces of the icosahedron to obtain a triangulation of 80 faces, 120 edges, and 42 vertices. Projecting radially to the sphere, we obtain a triangulation of the sphere. The vertices are expected to be approximately an optimal configuration of 42 points on the sphere. The process of the subdivision can be iterated to obtain arbitrarily large number of points on the sphere. In addition, after each subdivision, we may add an additional point, such as the centroid, to each triangle to increase the number of points. This modification of the algorithm does not lead to an approximately optimal configuration. On the other hand, by using the $\sqrt{3}$-subdivision algorithm (familiar from Geometric Modelling - see [Kobbelt 00]) followed by radial projection on the sphere, we may insert one additional point in each triangle while maintaining the approximate optimality property. We refer to the latter as $\sqrt{3}$ and to the former as midpoint subdivisions. They are variations on the same method. It is possible to start with configurations other than the icosahedron and proceed with the subdivision as before. This may lead to better configurations relative to the $\vartheta_{2}$ metric as shown later in the paper. A disadvantage of this method is that the set of $N$ to which the algorithm is applicable is very sparse. In fact, let $\nu_{S}(N)$ be the number of integers $l \leq N$ such that it is possible to distribute $l$ points on the sphere by subdivisions of the icosahedron. It is not difficult to show that $\nu_{S}(N) \simeq c \log N$.

\subsection{Polar Coordinates Subdivision}

This method has some similarity to the spiral algorithm of [Rakhmanov et al. 94]. For clarity of exposition, we first describe the method in a special case. Let $(\varphi, \theta)$ denote polar coordinates on the sphere with $-\frac{\pi}{2} \leq \varphi \leq \frac{\pi}{2}$ and $0 \leq \theta \leq 2 \pi$. We consider $(n-1)$ equally spaced latitudes $L_{j}$ corresponding to $\varphi=\phi_{j}=\frac{\pi j}{n}-\frac{\pi}{2}$ where $j=1, \ldots,(n-1)$. On the latitude $L_{j}$, we place $n_{j}$ equally spaced points, starting at $\theta=0$, where $n_{j}$ is given by

$$
n_{j}=\left\lfloor\frac{1}{2}+\sqrt{3} n \cos \phi_{j}\right\rfloor .
$$

On alternate latitudes, an obvious phase shift is imposed to make the configuration more symmetric. Together with the two poles, we obtain a configuration of points on $S^{2}$. This configuration is invariant under reflections relative to the equator and for this reason we refer to this special case as the symmetric polar coordinates method. Denoting by $\nu_{S P}(N)$ the number of $l \leq N$ which can be distributed on $S^{2}$ according to this algorithm, one shows easily that

$$
\nu_{S P}(N) \simeq C \sqrt{N}
$$


To overcome this undesirable feature of sparsity of $N$ to which the method is applicable, we make a perturbation of the algorithm. Instead of starting with the equator and distributing $n$ points evenly on it, we begin with a meridian parallel to and a distance $\epsilon>0$ from it. On the meridian, we evenly distribute $n$ points. Next, we proceed as in the special case of the symmetric polar coordinates method and distribute points on meridians parallel to the initial one. The distance of adjacent meridians and the number of points on each are determined by $n$ and the initial meridian. A shifting of points on alternate meridians is necessary to obtain approximately equilateral triangles. Notice that the poles are no longer necessarily part of the configuration of the points on the sphere. The actual implementation of the algorithm requires solving for $\epsilon$ and the number $n$ of points on the initial meridian, and we utilized the secant method with only few iterations. In this manner, we were able to more or less distribute any number of points on the sphere. More precisely, we say we have a gap of length $\lambda=j$ at $l$ if

$$
\min \left|l^{\prime}-l\right|=j
$$

where min taken relative to all integers $l^{\prime}$ for which we can distribute $l^{\prime}$ points on the sphere by this implementation. Table 1 shows the frequencies of gaps of various sizes for integers $\leq 4 \times 10^{5}$ in our implementation.

\begin{tabular}{|l|l|l|l|l|}
\hline Size of Gap & $\lambda=0$ & $\lambda=1$ & $\lambda=2$ & $\lambda \geq 3$ \\
\hline Frequency of Gaps & .9836 & .0118 & .0013 & .0033 \\
\hline
\end{tabular}

TABLE 1. Sizes and frequencies of gaps.

Note that for more than $98 \%$ of the integers $N \leq$ $4 \times 10^{5}$, we have realized distributions of $N$ points on $S^{2}$.

\section{NUMERICAL RESULTS}

To numerically study the distribution of points on a sphere via $\epsilon$-good sets, we considered all primes of the form $4 k+1$ less than 350 . For each $p$, we obtain $4(p+1)$ points on the sphere. Since the distribution of points depends on the choice of an initial point on the sphere, we calculated the metrics $\vartheta_{j}, j=1,2$, for five initial random points. It is clear that an inappropriate choice of an initial point (e.g., the point $(1,0,0)$ ) will lead to an "un-even" distribution of points. For random choices of initial point, there is little qualitative difference in the results. Figure 1 is the graph of $\log U_{N}$ versus $N$ for five different random initial points for all $p \equiv 1 \bmod 4$ and $\leq 349$. The bold face line is the average of the five values

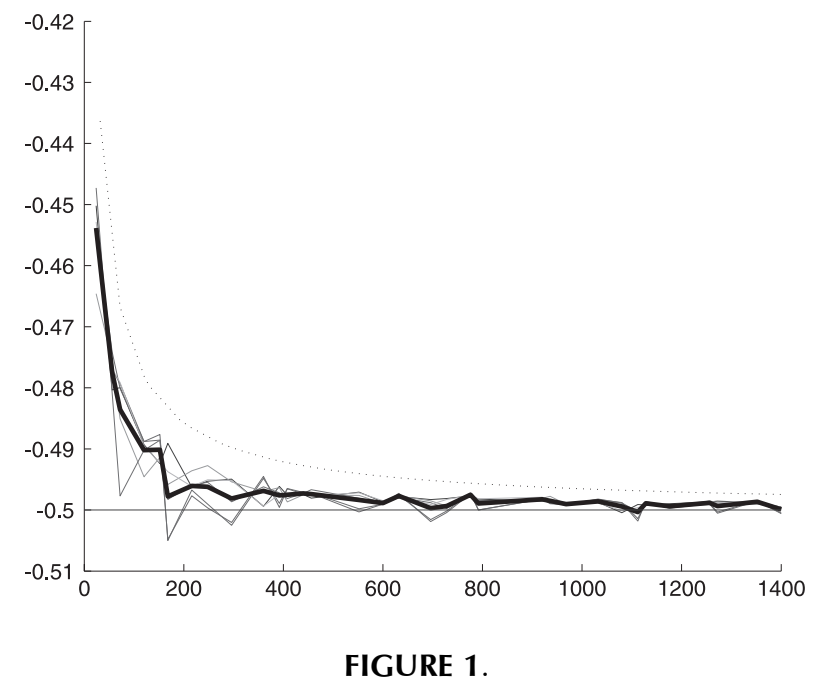

for $\log U_{N}$. The dotted line shows $\log U_{N}$ where the configuration is obtained via the lattice points method. It is clear that $\epsilon$-good sets do not provide satisfactory configurations for the distribution of points on the sphere relative to $\vartheta_{\circ}$. A similar conclusion is valid for $\vartheta_{1}$. Figure 2 shows the distribution of $N=552$ points $(p=137)$ on the sphere. The evident "clustering" resembles a similar phenomenon when points are chosen randomly according to the invariant measure.

Using the lattice points method, we generated up to $25,872=10\left(37^{2}+21^{2}+37.21\right)+2$ points on the sphere. Figure 3 shows $\vartheta_{1}$ versus the number of points for the lattice points method, midpoint and $\sqrt{3}$-subdivisions of the icosahedron, and polar coordinates methods. For graphical purposes and in view of the asymptotic expansion of $\mathcal{E}_{N}^{1}$, we normalized $\vartheta_{1}$ by division by the number of

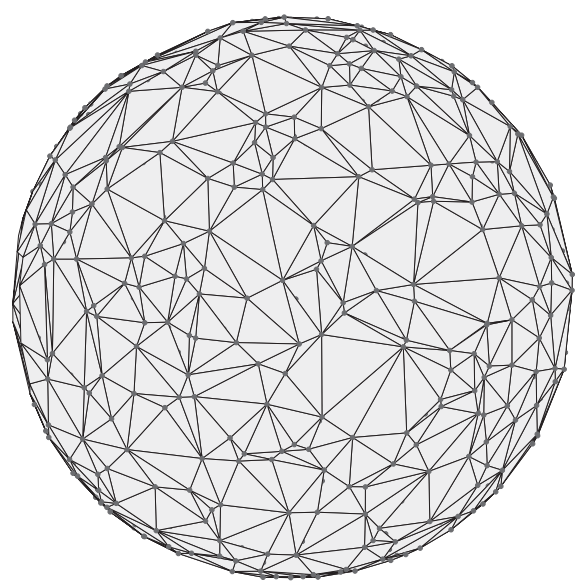

FIGURE 2. 


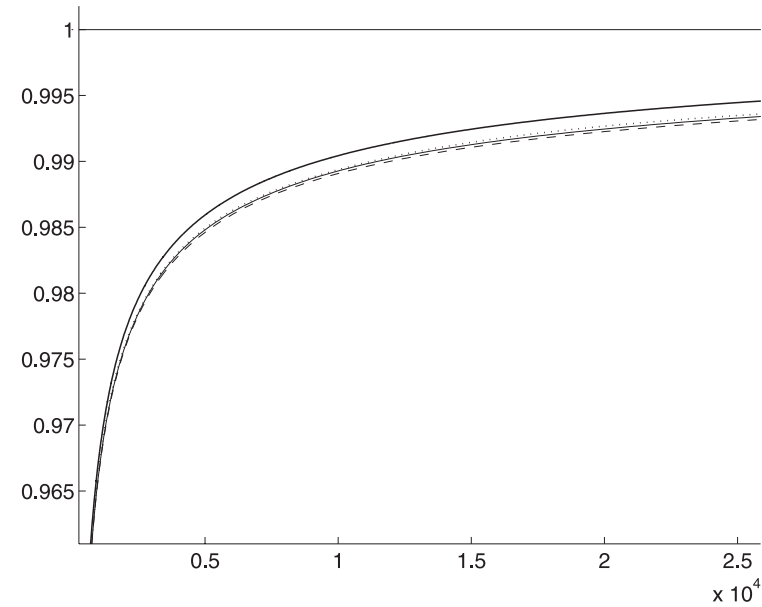

FIGURE 3. $\log U_{N}$.

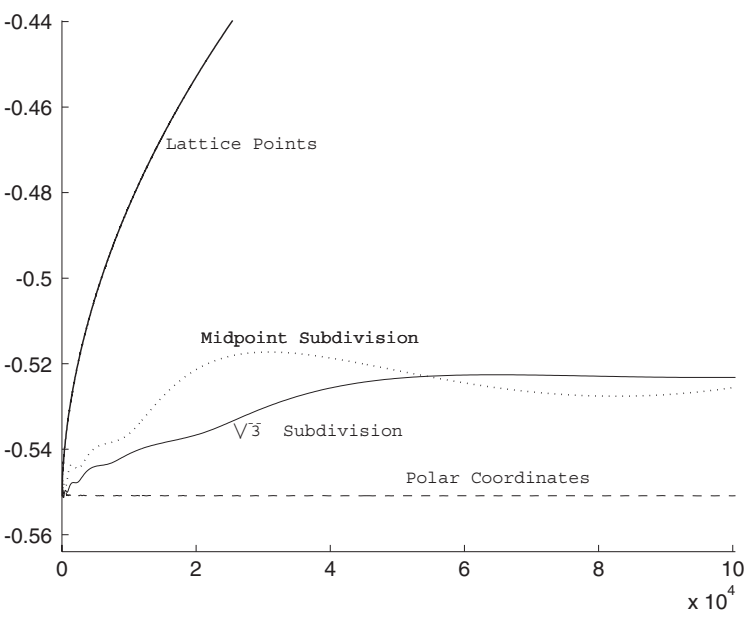

FIGURE 5. Calculation of $\xi_{1} \simeq \frac{\vartheta_{1}-\frac{N^{2}}{2}}{N^{\frac{3}{2}}}$.

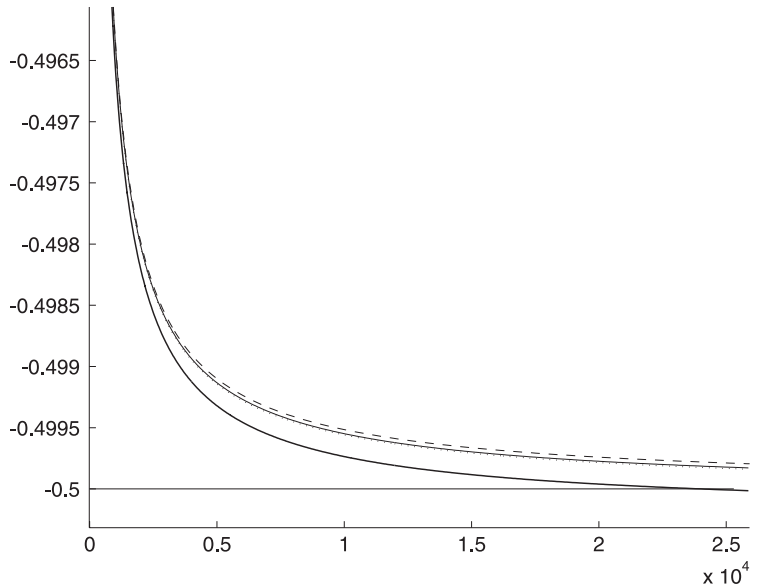

FIGURE 4. $\frac{\vartheta_{1}}{\left(\begin{array}{c}N \\ 2\end{array}\right)}$.

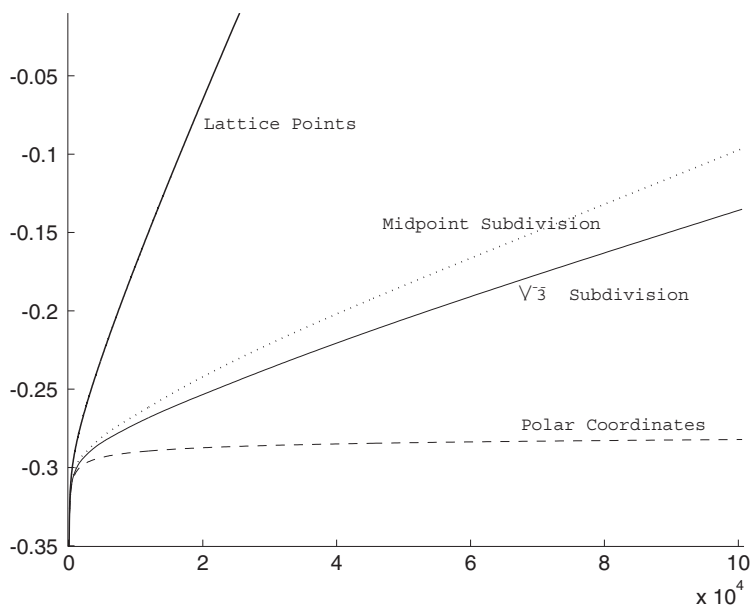

FIGURE 6. Calculation of $\xi_{\circ} \simeq \frac{\vartheta_{\circ}-\frac{N^{2}}{4}}{N \log N}$.

\begin{tabular}{|c|c|c|c|c|c|c|c|c|}
\hline No. of Points & 912 & 1082 & 1332 & 1482 & 2172 & 2432 & 2472 & 3332 \\
\hline Lattice & 400661 & 565705 & 860262 & 1066650 & 2302881 & 2891066 & 2987505 & 5444875 \\
\hline Polar & 400661 & 565704 & 860258 & 1066647 & 2302866 & 2891053 & 2987493 & 5444840 \\
\hline
\end{tabular}

TABLE 2. Comparison of Coulomb energy for the lattice points and polar coordinates methods after optimization.

pairs of points $\left(\begin{array}{c}N \\ 2\end{array}\right)$. Denoting the corresponding values of $\vartheta_{j}$ by $\vartheta_{j}^{A}, \vartheta_{j}^{M}, \vartheta_{j}^{\sqrt{3}}$, and $\vartheta_{j}^{P}$, respectively, we note the inequalities

$$
\vartheta_{1}^{A}>\vartheta_{1}^{M}>\vartheta_{1}^{\sqrt{3}}>\vartheta_{1}^{P}
$$

The number of points generated by the subdivision of the icosahedron grows exponentially fast with iterations, and therefore, the data for this method are very sparse. The curves in Figure 3 were obtained by spline interpolation. The number of configurations for the lattice points method was 796. No spline interpolation was necessary for the polar coordinates method since 24,486 configura- tions were available. The inequalities (4-1) raise doubts about the optimality conjecture in [Altschuler et al. 97]. In fact, we optimized $\vartheta_{1}$ (using a quasi-Newton method) for some configurations obtained via the polar coordinates for comparison with the lattice points method. The results (rounded off to the nearest integer), given in Table 2, demonstrate that the optimality conjecture of [Altschuler et al. 97] is incorrect. The fact that the optimized values for the two methods are close reflects the profusion of local minima for $\vartheta_{1}$.

Figure 4 shows the relative merits of the above methods relative to $\log U_{N}$ without the application of an op- 


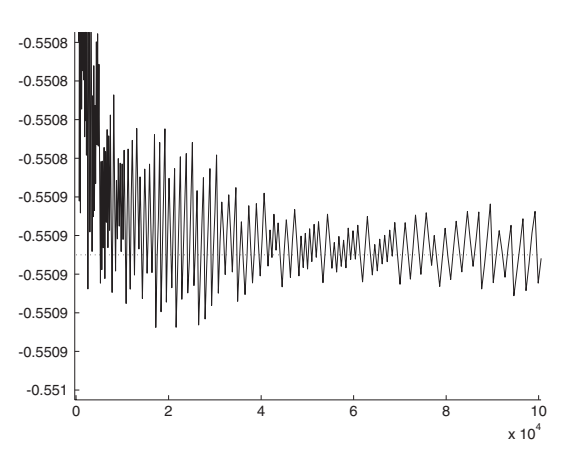

FIGURE 7. Calculation of $\xi_{1} \simeq \frac{\vartheta_{1}-\frac{N^{2}}{2}}{N^{\frac{3}{2}}}$.

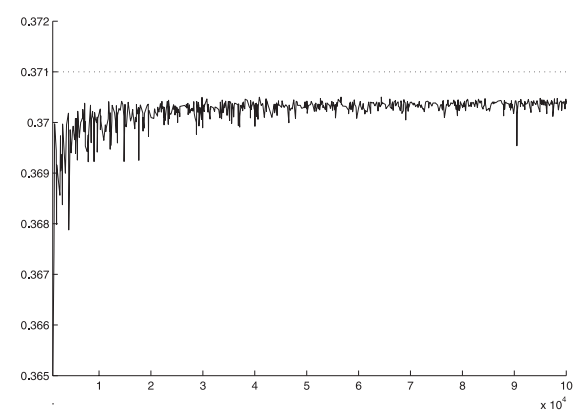

FIGURE 9. Calculation of $\eta_{\circ} \simeq \frac{\vartheta_{\circ}-\frac{N^{2}}{4}+\frac{N \log N}{4}}{N}$.

timization algorithm. In fact, we have the inequalities

$$
\vartheta_{\circ}^{A}>\vartheta_{\circ}^{M}>\vartheta_{\circ}^{\sqrt{3}}>\vartheta_{\circ}^{P}
$$

It appears that the polar coordinates method consistently provides more suitable configurations for points on the sphere. The advantage of the polar coordinates method over the lattice points and subdivision algorithms for both $\vartheta_{1}$ and $\vartheta_{\circ}$ is evident from Figures 5 and 6 .

For some moderate values of $N$, we applied a quasiNewton optimization algorithm to calculate $\vartheta_{\circ}^{A}$ and $\vartheta_{\circ}^{P}$ using the lattice points and polar coordinates methods for initial configurations. The differences in the values are generally in the decimal points only; they are given in Table 3.

From Figures 7 and 8 , the values of $\xi_{1}$ and $\eta_{1}$ may be approximately estimated, and we conjecture the follow-

\begin{tabular}{|l|l|l|l|l|l|l|l|l|}
\hline No. of Points & 912 & 1082 & 1332 & 1482 & 2172 & 2432 & 2472 & 3332 \\
\hline
\end{tabular}

\begin{tabular}{|l|c|c|c|c|c|c|c|c|}
\hline$\vartheta_{\circ}^{A}-\vartheta_{\circ}^{P}$ & -.087 & -.029 & .038 & .123 & .400 & .384 & .355 & .982 \\
\hline
\end{tabular}

TABLE 3. Comparison of $\vartheta_{\circ}^{A}$ and $\vartheta_{\circ}^{P}$ for the lattice points and polar coordinates methods after optimization.

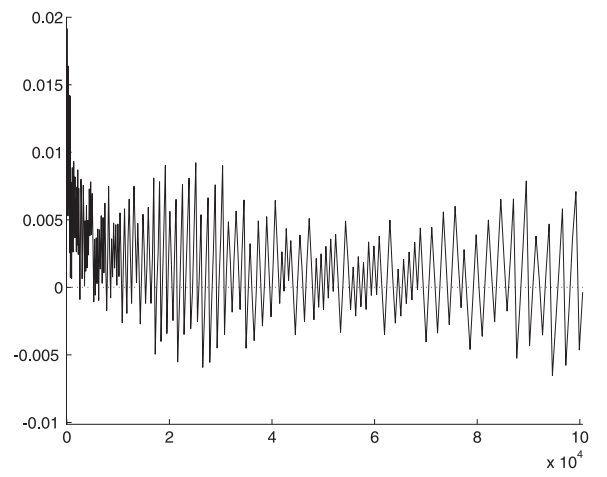

FIGURE 8. Calculation of $\eta_{1} \simeq \frac{\vartheta_{1}-\frac{N^{2}}{2}+\xi_{1} N^{\frac{3}{2}}}{N}$.

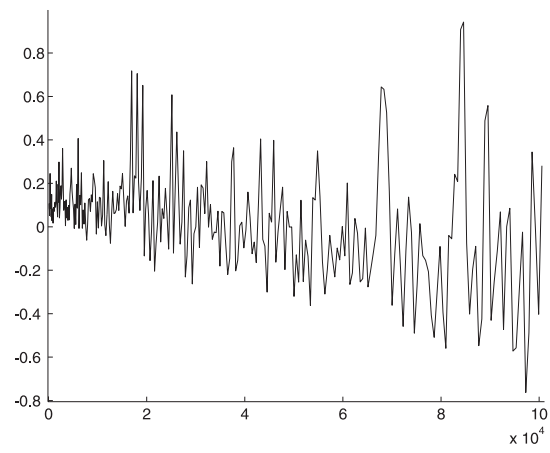

FIGURE 10. Coefficient of $\log N$ in $(4-4)$.

ing asymptotic expansion for $\vartheta_{1}$ :

$$
\vartheta_{1}=\frac{N^{2}}{2}-\alpha N^{\frac{3}{2}}+\beta N+O\left(N^{\frac{1}{2}}\right),
$$

with $\alpha \simeq .55$ and $0<\beta<<\alpha$. Similarly (see Figures 9 and 10), we conjecture the following asymptotic expansions for $\vartheta_{\circ}$ :

$$
\vartheta_{\circ}=\frac{N^{2}}{4}-\frac{N \log N}{4}+\gamma N+O(\log N),
$$

with $\gamma \simeq .37$.

An examination of the optimized versus nonoptimized (polar coordinates method) values for $\vartheta_{\circ}$ shows that the improvement in the value of $\vartheta_{\circ}$ is of the order of $\log N$. If the optimized values are indeed the global minima for those $N$ for which the optimization was carried out, then it is natural to conjecture that the polar coordinates configuration has indeed an error of the order of $\log N$ and the error in the polar coordinates method is quantified in $(2-5)$ with $C=2$.

The configurations of points according to the lattice points method has more symmetry than that of the polar coordinates method, however, the latter generally yields lower values for $\vartheta_{s}$. This may be interpreted as another 


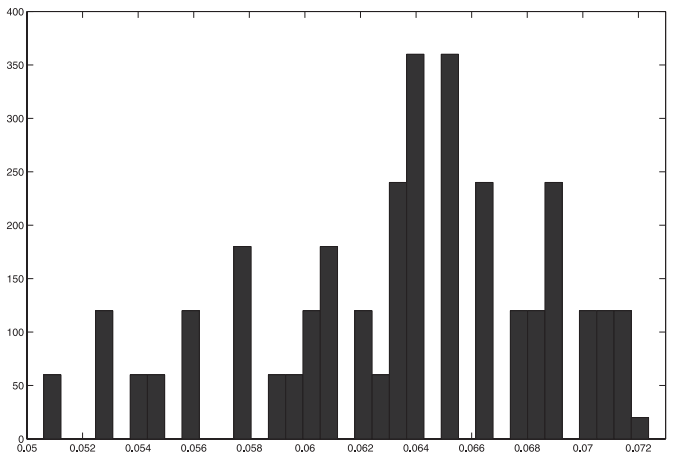

FIGURE 11. Histogram for minimum distances for lattice points method.

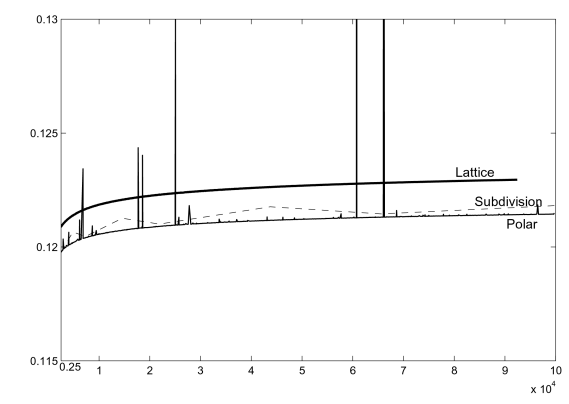

FIGURE 13. Calculation for $\frac{\vartheta_{2}}{N^{2} \log N}$ with different methods.

example of breakdown of symmetry in optimal configurations which is familiar in other applications as well (e.g., the central configuration in celestial mechanics). It also shows that in spite of the proximity of the values of $\vartheta_{\text {。 }}$ (or $\vartheta_{1}$ ) obtained by the lattice point or polar coordinates methods, they correspond to different local minima. A visual demonstration of the nature of $\vartheta_{1}$ for $N=2432$ is given in Figures 15 through 19. High-frequency (blue) areas are regions of low Coulomb energy and low-frequency (orange) areas are regions of high Coulomb energy. Red vertices are those with valence $\neq 6$ in the graph where each point is joined to its "nearest neighbors." For ease of comparison, all local energies have been scaled according to a universal scheme.

An examination of the lattice points and polar coordinates methods relative to $\vartheta_{\infty}$ generally favors the polar coordinates method. Figures 11 and 12 show typical histograms for the distribution of the minimum distances of the points on the sphere. Except for a few outlying points, the distribution of minimum distances by the polar coordinates method appears superior. This defect can

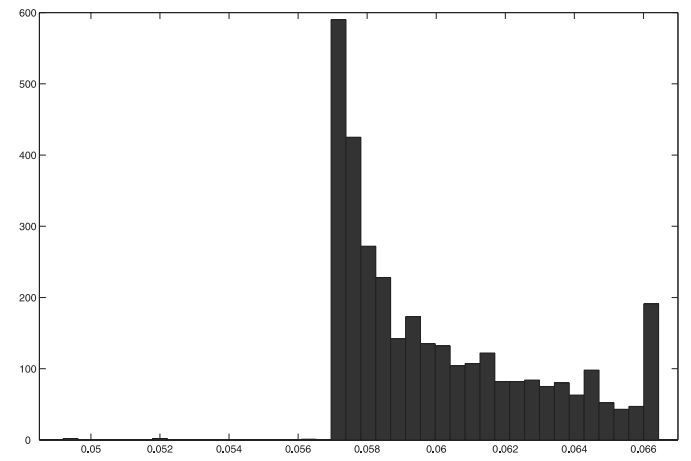

FIGURE 12. Histogram for minimum Distances for polar coordinates method.

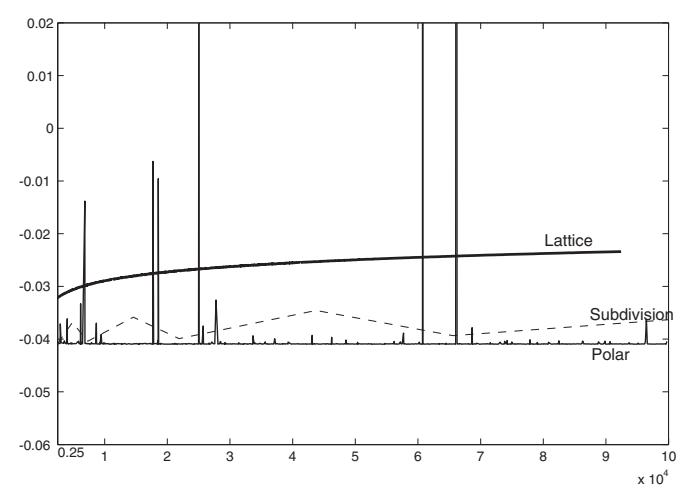

FIGURE 14. Calculation for $\xi_{2} \simeq \frac{\vartheta_{2}-\frac{1}{8} N^{2} \log N}{N^{2}}$ with different methods.

probably be rectified by a minor adjustment of the algorithm.

To better understand the distribution of points relative to $\vartheta_{2}$ metric, the lattice points, $\sqrt{3}$-subdivision, and polar coordinates methods were applied for the distribution of large numbers of points. Figure 13 shows that generally the polar coordinates method has lower value for $\vartheta_{2}$ than the subdivision or lattice points methods. There are a few exceptional cases where the polar coordinates method gives unreasonable values for $\vartheta_{2}$. After the elimination of these exceptional values of $N$, we still have configurations for the great majority of values of $N \leq 10^{5}$ with lower $\vartheta_{2}$ than other methods. In general, it is observed that there are subtle differences between distinct values for $N$, but it seems difficult to quantify these differences. In the application of the subdivision algorithm, we used as initial configurations the vertices of the icosahedron and 22 points with optimized configuration. The results show that subdivisions starting with the latter configuration give better results than those with the former. Figure 14 gives an estimate for 


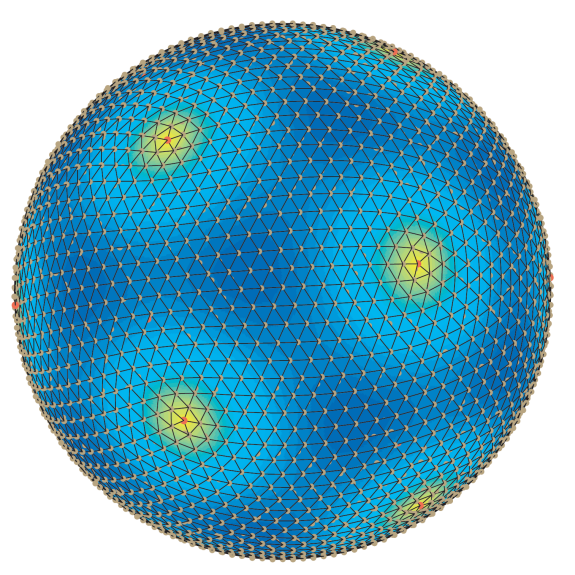

FIGURE 15. $\sqrt{3}$-subdivision prior to optimization.

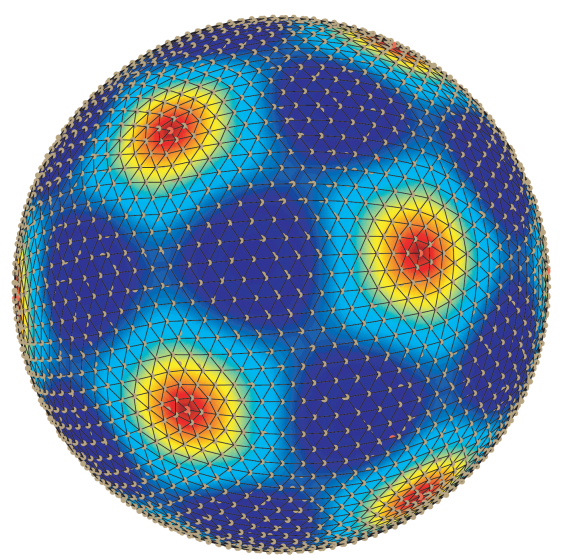

FIGURE 16. Lattice points method prior to optimization.

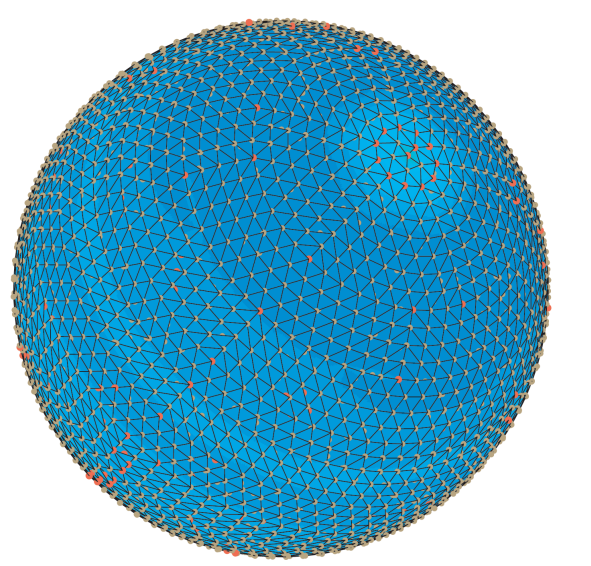

FIGURE 18. Polar coordinates method prior to optimization.
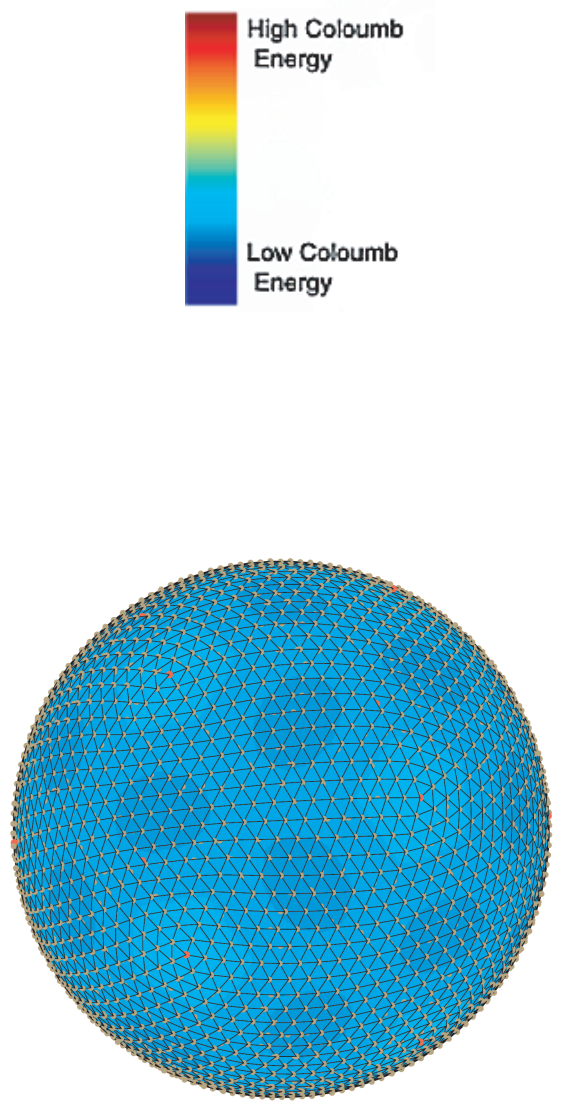

FIGURE 17. Initial lattice points configuration with Coulomb energy optimized.

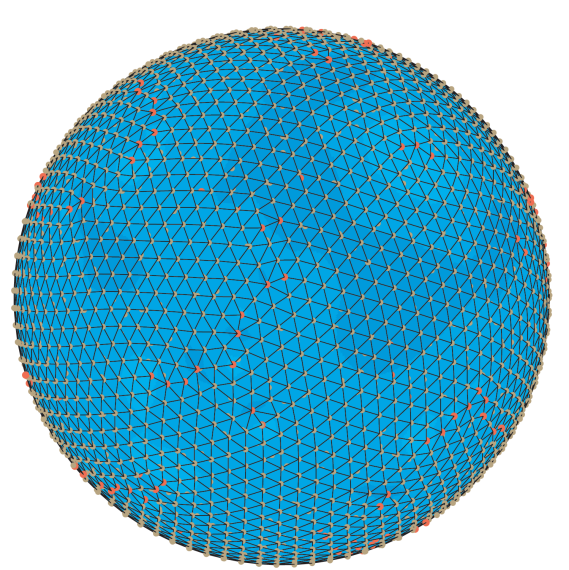

FIGURE 19. Initial polar coordinates configuration with Coulomb energy optimized. 
the value $\xi_{2}$ and we accordingly conjecture the following asymptotic expansion:

$$
\vartheta_{2} \simeq \frac{1}{8} N^{2} \log N-\delta N^{2}+o\left(N^{2}\right), \quad \text { where } \delta \simeq .04
$$

To each configuration of $N$ points, we assign a graph by joining each point to its "neighboring" ones for the appropriate definition of "neighbor." We used a standard triangulation method from computational geometry. The method of [Altschuler et al. 97] and the subdivision of the icosahedron have the obvious property that all but twelve vertices have valence 6 . In the polar coordinates method, we noticed that it is possible to have vertices with valences $4,5,6$, or 7 . Denoting the number of vertices of valence $j$ by $\mathcal{V}_{j}$, we noticed that

$\mathcal{V}_{4}=0$ for sufficiently large $N, \quad \mathcal{V}_{5}=\mathcal{V}_{7}+12=O\left(N^{\frac{1}{2}}\right)$

For each vertex $v$ of a triangulation of compact surface $M$ without boundary, let $\kappa_{v}=6-\mathcal{V}(v)$ where $\mathcal{V}(v)$ denotes the valence of the vertex $v$. One may reasonably call $\kappa_{v}$ the discrete curvature of the given triangulation at the vertex $v$. Then we have the following discrete version of the Gauss-Bonnet theorem for surfaces:

$$
\sum_{v} \kappa_{v}=6 \chi(M)
$$

where $\chi(M)$ denotes the Euler characteristics of $M$. The proof of this identity is by a straightforward substitution in the definition of $\chi(M)$ and the simplest case of the Dehn-Sommerville relations, namely,

$$
2 e=3 f
$$

where the $e$ and $f$ denote the number of edges and faces of the triangulation, respectively. A consequence of (4-5) is that if there are only vertices with valences 5,6 , and 7 , then $\mathcal{V}_{5}=\mathcal{V}_{7}+12$ for any triangulation of the sphere. It is immediate that the $S^{2}$ admits of triangulations with constant discrete curvature $\kappa=1,2$, or 3 and the torus admits of triangulations with zero discrete curvature. For surfaces $M_{g}$ of genus $g \geq 2$, there are triangulations of constant discrete curvature -1 , and for most $g \geq 5$, we have constructed triangulations of $M_{g}$ with constant discrete curvature -2 . Generally, there is a profusion of distinct triangulations for given constant discrete curvature $\kappa<0$ and $M_{g}$ (when it exists), but its quantification seems quite difficult. A generalization of the notion of discrete curvature $\kappa_{v}$ to higher dimensions is possible. There is also empirical evidence that the distribution of the eigenvalues of the dual graphs (i.e., 1-skeletons of the subdivision dual to the given triangulation), which are 3-regular graphs, do in fact reflect some of the metric properties of the configuration. For certain sequences of configurations obtained by subdivisions, the empirical distributions of the eigenvalues converge. The nature of the limiting measure, whether it is pure point, singular, or absolutely continuous, is difficult to determine; empirically, it seems to reflect the metric properties of the method of subdivision. These issues will be elaborated on elsewhere.

A preprint of this article can be found at http://math.ipm.ac.ir/scc/publications.htm.

\section{ACKNOWLEDGMENTS}

The second named author (Shahshahani) wishes to thank Persi Diaconis for helpful conversations on the problem of distributing points on the sphere.

\section{REFERENCES}

[Altschuler et al. 97] E. W. Altschuler et al. "Possible Global Minimum Lattice Configurations for Thomson's Problem of Charges on a Sphere." Physical Review Letters 78 (1997), 2681-2685.

[Greengard and Rokhlin 97] L. Greengard and V. Rokhlin. "A New Version of the Fast Multipole Method for the Laplace Equation in Three Dimensions." Acta Numerica (1997), 229-269.

[Kobbelt 00] L. Kobbelt. " $\sqrt{3}$ Subdivision." In Proceedings of SIGGRAPH 2000, Computer Graphics Proceedings, Annual Conference Series, edited by Kurt Akeley, pp. 103-112, Reading, MA: Addison-Wesley, 2000.

[Kuijlaars and Saff 98] A. B. J. Kuijlaars and E. B. Saff. "Asymptotics for Minimal Discrete Energy on the Sphere." Trans. Amer. Math. Soc. 350 (1998), 523538.

[Rakhmanov et al. 94] E. A. Rakhmanov, E. B. Saff, and Y. M. Zhou. "Minimal Discrete Energy on the Sphere." Math. Research Letters 1 (1994), 647-662.

[Sarnak 90] P. Sarnak. Some Applications of Modular Forms. Cambridge, UK: Cambridge University Press, 1990.

[Shub and Smale 93] M. Shub and S. Smale. "Complexity of Bezout's Problem III: Condition Number and Packing." Jour. of Complexity 9 (1993), 4-14.

[Smale 00] S. Smale. "Mathematical Problems for the Next Century." In Mathematics: Frontiers and Perspectives, edited by Arnold, Atiyah, Lax, and Mazur. Providence, RI: Amer. Math. Society, (2000). 
[Tsuji 75] T. Tsuji. Potential Theory in Modern Function Theory. New York: Chelsea Publishing Company, 1975.
[Thomson 04] J. J. Thomson. "On the Structure of the Atom: ...." Philosophical Magazine and Journal of Science Sixth Series (1904), 237-265.

Ali Katanforoush, Institute for Studies in Theoretical Physics and Mathematics, P.O. Box 19395-5746, Tehran, Iran (katanfor@ipm.ir)

Mehrdad Shahshahani, Institute for Studies in Theoretical Physics and Mathematics, P.O. Box 19395-5746, Tehran, Iran (mehrdads@ipm.ir or sl2z@aol.com)

Received June 28, 2002; accepted in revised form April 24, 2003 